\title{
Oncologic validity of minimally invasive lobectomy for early stage lung cancer
}

\author{
Todd L. Demmy, Sai Yendamuri \\ Department of Thoracic Surgery, Roswell Park Cancer Institute, Buffalo, NY, USA \\ Correspondence to: Todd L. Demmy, MD. Professor, Department of Thoracic Surgery, Roswell Park Cancer Institute, Elm and Carlton Streets, \\ Buffalo, NY 14263, USA. Email: todd.demmy@roswellpark.org. \\ Provenance: This is an invited article commissioned by the Academic Editor Hexiao Tang (Division of Thoracic Surgery, Massachusetts General Hospital, \\ Harvard Medical School, Boston, MA, USA; Division of Thoracic Surgery, Zhongnan Hospital of Wuhan University, Wuhan, China). \\ Comment on: Yang CJ, Kumar A, Klapper JA, et al. A National Analysis of Long-term Survival Following Thoracoscopic Versus Open Lobectomy for \\ Stage I Non-small-cell Lung Cancer. Ann Surg 2019;269:163-71.
}

Submitted Jul 12, 2019. Accepted for publication Sep 11, 2019.

doi: $10.21037 /$ jtd.2019.09.45

View this article at: http://dx.doi.org/10.21037/jtd.2019.09.45

\section{Introduction}

Minimally invasive pulmonary lobectomy was first performed decades ago and was adopted quite slowly compared to other common major laparoscopic or thoracoscopic procedures. The reasons behind slow adoption were complex, multifactorial and beyond the scope of this article, but concerns about oncologic validity have been cited as concerns by traditional open surgeons. A recent article by Yang and colleagues adds to a growing body of works serving to dispel these concerns (1). In this commentary, we will discuss this paper as well as supplement our previously published arguments regarding the oncologic validity of minimally invasive lobectomy (MIL) with more recent research (2).

Randomized comparisons are desirable but extremely hard to attract volunteers to agree to be allotted to more invasive procedures. It took a very high-volume consortium in China to achieve recruitment (compared to axillary thoracotomy) and long-term results are pending (3). Long term survival data are pending but the short-term results replicate the preponderance of evidence showing fewer complications and faster recovery (3). A number of meta-analyses including a recent systematic review on the optimal approach to thoracoscopic lobectomy demonstrate equivalent or favorable outcomes with MIL (4). In the past several years, there has been more written about longer term outcomes with MIL. Table 1 summarizes other work since our last review on long-term survival (2).

\section{Pros and cons of large database reviews}

The article by Yang and colleagues uses results from the National Cancer Database (NCDB) to help us understand long-term survival (1). These resources are powerful because of the large sample sizes that allow detection of small effects and ability to match many cases to control for bias. In addition, as these databases collate data not just from quaternary care centers, but from other treating hospitals on the frontline of healthcare delivery, it is useful to understand the impact of new surgical approaches as they are adopted more broadly. However, there are many limitations as well because selection bias was inherent with the introduction of MIL initially accepted only for small peripheral tumors (13). Central tumors in lymph node rich basins requiring complex resection techniques were approached more by open surgery but a variable easily defining centrality does not exist in most current data sets. One European center was able to control for this centrality bias in its prospective database (14). In addition, important dependent variables are not captured by databases such as the NCDB. Also, in the discussion of long-term oncologic outcome, the relevant outcome is recurrence free survival or disease-specific survival, both of which are absent in the NCDB. Hospital selection bias is likely and when every hospital is captured in a state such as the New York Statewide Planning and Research Cooperative System covariates that trend with MIL become more evident like female sex, lower comorbidity index, insurance, older age, 


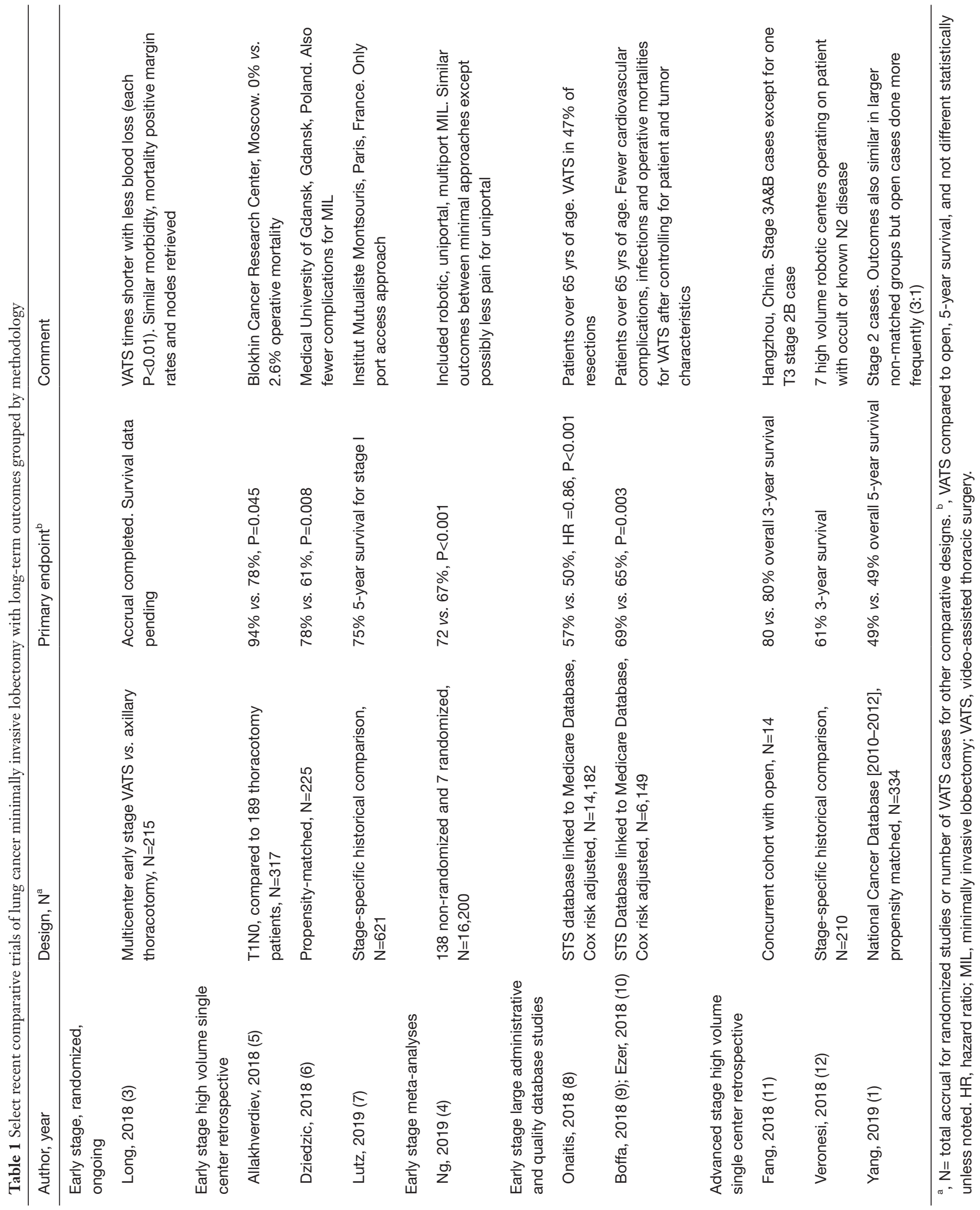


surgery in recent year, nonteaching hospital, and higher annual lung surgery volume (15).

\section{Technician versus the technology}

An important point not been emphasized much in the debate about MIL is that significant upgrades in optics and instruments have been made during the time these databases aggregated patients and continue to evolve $(16,17)$. Early on, many excellent surgeons were frustrated with the ability to see the anatomy and manipulate it compared with open approaches. A mechanism of inferior technology impairing surgeon intentions and thereby outcomes was very plausible. Now that high definition cameras and better articulated tools including robotic platforms are very accessible, surgeons can replicate and sometimes exceed what they were trained (or inclined) to do before MIL. This is exemplified by research showing that oncologic concerns of upstaging and quality of lymphatic dissection are associated more with the experience of the program than the technology or the approach, per se (18). Current technological trends such as enhanced optics (including augmented reality, near infrared imaging, and very high pixel density) and new robotic or robotic emulating handheld instruments will approach or even surpass open technical capabilities (19).

Other issues limit surgeon interest in approach effect on early stage disease. For one, our surgical investigator predecessors tended to be interested more in effects resulting from operative anatomic changes than the exposure used to achieve them. Visual inspection that a new technology or exposure approach achieved a result comparable to previous methods was enough to justify a modest non-randomized series and consider substitution or adoption if there were no medium or long-term variations from expected classic outcomes. If an update to imaging software or therapeutic radiation medicine equipment generates ostensible improvement to anatomic clarity or ablative precision, subjecting large numbers of patients to comparisons with inferior technology beyond limited safety trials becomes ethically problematic. Similarly, expensive phase III drug research generally is warranted only by nonrandomized phase 2 signaling a therapeutic benefit.

Special populations are particularly well suited to less invasive approaches. Frail patients such as the elderly gain better access to aggressive surgical procedures if thoracotomy can be avoided and represented the vanguard at some centers (20). Arguably, comorbid medical problems might obscure higher local recurrence rates; however, research indicates that avoidance of non-oncologic mortality from various complications is one of the strongest arguments for MIL even for aggressive diseases like lung cancer (21).

Surgeons are not the only professionals tasked with introducing new technologies designed to improve safety and results. Architects and engineers do this by using stress testing and computer models on new materials. Likewise, some thoracic surgeons are "stress testing" our minimally invasive approaches on patients with higher stage lung cancer. We have not seen reports of accelerated local recurrences that would be expected intuitively if MIL was fundamentally an inferior oncologic operation. Table 1 adds to the advanced resection studies listed in our previous review that compare similarly or favorably to open studies. The lack of a "failure" signal for patients with aggressive extensive stage tumors reduces enthusiasm for pursuing research for recurrence in stage 1 populations.

\section{Research priorities}

Because of plausible hypotheses that impaired exposures would adversely affect oncologic outcomes and extra resources needed to establish MIL at busy high-performing open surgery centers, it was reasonable to study surgical approach. Before MIL, innovations in open oncologic surgical approaches typically gained acceptance with similar or less controversy. The adequacy of anatomic dissection (like nodal sampling versus radical lymphadenectomy) or sublobar resection remain questions of interest. Now that we have results of Yang and so many other trials without negative signals, it seems time to move past the simple question of approach as the research focus. It can be revisited if concerns with long-term oncologic outcomes emerge unexpectantly in different research efforts or in the study of special populations where approach is more likely to generate a measurable effect (like advanced stage patients). Once such a signal is detected, then it will be necessary not only to design a study to analyze an outcome problem but also test a hypothesis that will explain the adverse outcomes. Minimally invasive operations may have longer operative times and perhaps tumor manipulations that could be suboptimal with small instruments. Apart from these, it is difficult to conceive of mechanisms by which more traumatic incisions and the bleeding and pain associated with them will benefit patients.

Without easier accessibility to randomized research, our databases need to evolve to answer scientific questions 
of future relevance. For this to happen, there needs to be much more flexible, dynamic, granular data and formatting that allows for testing hypotheses not predicted by the traditional database organizing committees. This will require a great expansion of optional data fields with participating programs empowered to enter structured data if they have the resources to do so. Surgical specialty societies might promote a uniform description rubric for operative reports to allow for attachment of deidentified text files that could be searched by artificial intelligence engines. Then, for example, important questions requiring large sample sizes like whether general anesthetic duration or order of vascular division during lobectomy affects survivals could be then tested with big databases.

It is reasonable to expect that data integration that is currently underway to deal with the problem of duplicate storage and lack of uniform accessibility to radiology images could be leveraged to make patient participation in an outcomes database more dynamic. That is, centralized administrative databases like NCDB could have metadata links back to healthcare records and even images that allow accessing data points that become meaningful over time. As mentioned, one of the major limitations of the Yang article and any large database study is that there is natural selection bias against central tumors because the associated difficulty with dissection made this a relative contraindication for MIL. Unfortunately, centrality is difficult to define just as it often challenging to determine preoperatively whether anatomic structures are invaded or simply effaced. The ability to link imaging back to the record would be useful to quantify centrality for propensity matching, for database quality control, and ultimately allow merging of radiomic, genomic, and clinical data points.

Currently databases like NCDB are highly controlled, constrained by financial resources and centralized bureaucracies, and change slowly unlike the human beings that they study. While controlling for variability is essential to scientists in general (and surgeons in particular), it may not be a practical solution for our future as surgical investigators. It is also inconsistent with the expectations, practices and online social interconnectivity of our patients. It seems inevitable that a hybrid approach to studying clinical outcomes will emerge. Current infrastructures for database abstraction at the institution level will be necessary to accurately and consistently document events that occur perioperatively. After a patient leaves that environment, better data might be obtained by other health care providers who interact directly with the patient or even the participant themselves.
Authors have attempted to validate hypotheses generated by much smaller cohorts at their own institutions by using larger datasets. If databases like the NCDB could authorize optional collection of expanded data forms that could reside centrally or at the local institutional level, then it could become a powerful engine of investigator collaboration. At relatively low cost, consortiums could emerge quickly around their existing core infrastructures by backloading data to test new hypotheses or validate or refute the publications of others.

This will require a disruptive change to the governance of database and human research protections organizations. Yet this change seems inevitable because of electronic medical record integration across all healthcare environments necessary to eliminate handoff errors and duplication of services. It is reasonable to expect that patients (as they do now) will trade privacy of their personal content to achieve convenience of an integrated health care service that will probably be offered to them free of charge and perhaps with rewards for validating data. Researchers will be able to access the record of the patient for a fee to an organization (akin to Facebook) that has provided that service. All in all, that fee will probably be less than the salary of a research assistant to make phone calls.

This opportunity is under development indirectly or directly by companies like Amazon, Google, Apple, and Microsoft that have multi-billion-dollar partnerships to enhance services in the medical space that ultimately will require research mechanisms to prove their worth.

In summary, it is high time for thoracic surgeons to move past testing things like surgical approach given paucity of evidence suggesting that open surgery benefits patient longevity or human desire for less body invasion or lifestyle disruption. Instead, physician investigators need to lead or partner with industry in the effort to enhance cooperative database design. In this way, collaborative group science will improve and the surgeon's role in designing meaningful, mechanistic-based randomized trials for the diseases we treat will be enhanced.

\section{Acknowledgments}

None.

\section{Footnote}

Conflicts of Interest: TL Demmy: Medtronic. S Yendamuri has no conflicts of interest to declare. 
Ethical Statement: The authors are accountable for all aspects of the work in ensuring that questions related to the accuracy or integrity of any part of the work are appropriately investigated and resolved.

\section{References}

1. Yang CJ, Kumar A, Klapper JA, et al. A National Analysis of Long-term Survival Following Thoracoscopic Versus Open Lobectomy for Stage I Non-small-cell Lung Cancer. Ann Surg 2019;269:163-71.

2. Demmy TL, Yendamuri S, D'Amico TA, et al. Oncologic Equivalence of Minimally Invasive Lobectomy: The Scientific and Practical Arguments. Ann Thorac Surg 2018;106:609-17.

3. Long H, Tan Q, Luo Q, et al. Thoracoscopic Surgery Versus Thoracotomy for Lung Cancer: Short-Term Outcomes of a Randomized Trial. Ann Thorac Surg 2018;105:386-92.

4. Ng CSH, MacDonald JK, Gilbert S, et al. Optimal Approach to Lobectomy for Non-Small Cell Lung Cancer: Systemic Review and Meta-Analysis. Innovations (Phila) 2019;14:90-116.

5. Allakhverdiev A, Davydov M, Akhmedov P. Thoracoscopic lobectomy with mediastinal lymph node dissection as a standard surgery for T1-2N0M0 non-small cell lung cancer (>300 surgeries experience). Ann Med Surg (Lond) 2018;35:169-72.

6. Dziedzic R, Marjanski T, Binczyk F, et al. Favourable outcomes in patients with early-stage non-small-cell lung cancer operated on by video-assisted thoracoscopic surgery: a propensity score-matched analysis. Eur J Cardiothorac Surg 2018;54:547-53.

7. Lutz JA, Seguin-Givelet A, Grigoroiu M, et al. Oncological results of full thoracoscopic major pulmonary resections for clinical Stage I non-small-cell lung cancer. Eur J Cardiothorac Surg 2019;55:263-70.

8. Onaitis MW, Furnary AP, Kosinski AS, et al. Prediction of Long-Term Survival After Lung Cancer Surgery for Elderly Patients in The Society of Thoracic Surgeons General Thoracic Surgery Database. Ann Thorac Surg 2018;105:309-16.

9. Boffa DJ, Kosinski AS, Furnary AP, et al. Minimally Invasive Lung Cancer Surgery Performed by Thoracic Surgeons as Effective as Thoracotomy. J Clin Oncol 2018;36:2378-85.

10. Ezer N, Kale M, Sigel K, et al. Outcomes after Videoassisted Thoracoscopic Lobectomy versus Open Lobectomy for Early-Stage Lung Cancer in Older Adults. Ann Am Thorac Soc 2018;15:76-82.
11. Fang L, Wang L, Wang Y, et al. Video assisted thoracic surgery vs. thoracotomy for locally advanced lung squamous cell carcinoma after neoadjuvant chemotherapy. J Cardiothorac Surg 2018;13:128.

12. Veronesi G, Park B, Cerfolio R, et al. Robotic resection of Stage III lung cancer: an international retrospective study. Eur J Cardiothorac Surg 2018;54:912-9.

13. Swanson SJ, Herndon JE 2nd, D'Amico TA, et al. Videoassisted thoracic surgery lobectomy: report of CALGB 39802--a prospective, multi-institution feasibility study. J Clin Oncol 2007;25:4993-7.

14. Decaluwé H, Stanzi A, Dooms C, et al. Central tumour location should be considered when comparing N1 upstaging between thoracoscopic and open surgery for clinical stage I non-small-cell lung cancer. Eur J Cardiothorac Surg 2016;50:110-7.

15. Wolf A, Liu B, Leoncini E, et al. Outcomes for Thoracoscopy Versus Thoracotomy Not Just Technique Dependent: A Study of 9,787 Patients. Ann Thorac Surg 2018;105:886-91.

16. Wahba R, Datta RR, Hedergott A, et al. 3D vs. $4 \mathrm{~K}$ Display System - Influence of "State-of-the-art"-Display Technique On Surgical Performance (IDOSP-Study) in minimally invasive surgery: protocol for a randomized cross-over trial. Trials 2019;20:299.

17. Dunning J. Disruptive technology will transform what we think of as robotic surgery in under ten years. Ann Cardiothorac Surg 2019;8:274-8.

18. Krantz SB, Lutfi W, Kuchta K, et al. Improved Lymph Node Staging in Early-Stage Lung Cancer in the National Cancer Database. Ann Thorac Surg 2017;104:1805-14.

19. Nelson DB, Mehran RJ, Mitchell KG, et al. RoboticAssisted Lobectomy For Non-Small Cell Lung Cancer: A Comprehensive Institutional Experience. Ann Thorac Surg 2019;108:370-6.

20. Demmy TL, Curtis JJ. Minimally invasive lobectomy directed toward frail and high-risk patients: a case-control study. Ann Thorac Surg 1999;68:194-200.

21. Hristov B, Eguchi T, Bains S, et al. Minimally Invasive Lobectomy Is Associated With Lower Noncancer-specific Mortality in Elderly Patients: A Propensity Score Matched Competing Risks Analysis. Ann Surg 2018. [Epub ahead of print].

Cite this article as: Demmy TL, Yendamuri S. Oncologic validity of minimally invasive lobectomy for early stage lung cancer. J Thorac Dis 2019;11(10):E163-E167. doi: 10.21037/ jtd.2019.09.45 